\title{
Performance Analysis of Selected Error Control Protocols in Wireless Multimedia Sensor Networks
}

\author{
M. Yousof Naderi \\ Hamid R. Rabiee \\ Mohammad Khansari \\ Advanced Information and Communication Technology Center (AICTC) \\ Department of Computer Engineering \\ Sharif University of Technology \\ Tehran, Iran \\ Email: mynaderi@ce.sharif.edu \{rabiee,khansari\}@sharif.edu
}

\begin{abstract}
Error control is an important mechanism for providing robust multimedia communication in wireless sensor networks. Although there have been several research works in analysis of error control mechanisms in wireless multimedia networks and wireless sensor networks, but none of them are directly applicable to the wireless multimedia sensor networks (WMSNs) which has resource and performance constraints of WSNs as well as QoS requirements of multimedia communications. In this paper, we comprehensively evaluate the performance of several error control mechanisms in WMSNs. The results of our analysis provide an extensive comparison between automatic repeat request (ARQ), forward error correction (FEC), and hybrid FEC/ARQ error control mechanisms in terms of frame loss rate, frame peak signal-tonoise ratio (PSNR), and energy efficiency.
\end{abstract}

\section{INTRODUCTION}

In recent years, there has been an increasing interest in applications of wireless sensor networks (WSNs). Applications such as real-time object tracking, source localization, multimedia surveillance, advanced healthcare delivery, and industrial process control [1][2][3] which all require gathering of information in the form of multimedia such as audio, image and video; and thus necessitate efficient and reliable multimedia communication in WSNs.

The multimedia content in sensor networks should be delivered with predefined levels of quality of service (QoS) under resource and performance constraints such as bandwidth, energy, and delay. These constraints limit the extent to which QoS requirements can be guaranteed. Although a high compression ratio makes multimedia applications suitable for low bit-rate wireless channels, the compressed multimedia stream become more vulnerable to transmission errors due to predictive coding. Moreover, low power communication constraints of sensor nodes worsen the effects of wireless channel errors and require energy-efficient communication protocols in order to achieve application objectives, while delivery of multimedia streams may be an energy consuming task. All of these challenges necessitate energy efficient and reliable error control schema for QoS multimedia communication over multi-hop WSNs.
The well-known error control mechanisms to deal with wireless transmission errors in multimedia streaming applications include forward error correction (FEC), automatic repeat request (ARQ), and hybrid FEC/ARQ schemas. This paper investigates the performance evaluation of error control schemes for real-time multimedia communication scenarios. To this end, we perform a wide range of simulations and provide strengths and shortcomings of different error control mechanisms for multimedia streaming over multi-hop WSNs. This analysis enables a comparison of ARQ, wireless link-layer FEC, and hybrid FEC/ARQ schemas based on QoS and energy performance metrics in WMSNs. More specifically, the analysis of frame loss rate, PSNR, and energy efficiency is preformed by considering MicaZ video sensor nodes. To the best of our knowledge, this is the first work that comparatively evaluates the performance of conventional error control schemas for real-time multimedia communications in sensor networks.

The rest of this paper is organized as follows: In Section II, we describe the considered system model for WMSN channel, and energy consumption. Section III describes our simulation methodology and presents our performance evaluation results. Finally, the paper is concluded in Section IV.

\section{SYSTEM MODEL}

\section{A. Channel Model}

In order to capture bit-level errors, we model WMSN channel with a two-state Markov chain called Gilbert-Elliott channel [4].

It has been demonstrated that this is a good approximation of the error characteristics in a wireless channel [5]. Figure 1 illustrates a state diagram for a 2-state Markov model of Gilbert-Elliott channel. In particular, this model abstracts bursty error distribution with one state representing a heavy error rate (bad state) with a short interval, and the other representing a longer interval of light error (good state). Moreover, each state having an associated error probability and state transition probabilities can be derived from the experimental channel data. 


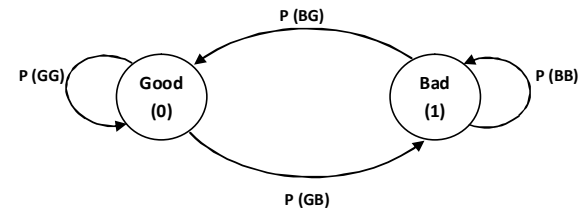

Figure 1. Markov model for Gillber-Elliot channel

The stationary probabilities of being in good and bad state are given by

$$
\pi_{G}=\frac{p_{B G}}{p_{B G}+p_{G B}} \quad \pi_{B}=\frac{p_{G B}}{p_{B G}+p_{G B}}
$$

Where $\mathrm{P}(\mathrm{GB})$ is the probability of the state transiting from a good state to a bad state, and $\mathrm{P}(\mathrm{BG})$ is the transition from a bad state to a good state. Based on the channel model that we just described, we can express the average bit error probability of the WMSN channel as follows:

$$
p=p_{G} \pi_{G}+p_{B} \pi_{B}
$$

\section{B. Energy Consumption Model}

We use an energy model based on the MicaZ Motes [6] in order to model the power consumption of a wireless multimedia node. This hardware is widely used in sensor network research and it is suitable for WMSNs because of its data rate and energy efficiency [3].

According to our model, a node may consume energy during the reception, transmission, decoding, and encoding of transmitted/received packets as well as in the idle state. The power consumption during the transmit mode, the receive mode, and the idle mode, are denoted by $P_{r}, P_{t}$ and $P_{i}$ respectively. If a sensor node spends $\mathrm{T}$ seconds transmitting or receiving a packet, the energy consumption can be computed as: $\mathrm{E}_{T x}(\mathrm{~T})=\mathrm{P}_{t} T$ or $\mathrm{E}_{R x}(\mathrm{~T})=\mathrm{P}_{r} T$ respectively. The energy dissipated during an idle listening period of $\mathrm{T}$ seconds is also calculated as: $E_{I}(T)=P_{i} T$. Note that the major overhead of FEC codes is the energy consumption for decoding and encoding of packets. Since it is well known that the energy consumed at the FEC encoder is negligible [7][8], we only consider the decoding energy of FEC block codes in our simulations.

In particular, the amount of power that multimedia sensor nodes require to decode RS codes is computed based on the total codeword length and the FEC code length[7]. Hence, first the latency of decoding for a $\mathrm{RS}(\mathrm{u}, \mathrm{w})$ is calculated and then the decoding energy consumption is computed using current and supply voltage of the processor. Note that RS $(\mathrm{u}, \mathrm{w})$ means $\mathrm{w}$ data bytes and (u-w) correction RS bytes where (u-w) correction bytes can restore $(\mathrm{u}-\mathrm{w}) / 2$ corrupt bytes. According to [8][9], the decoding latency for a $\mathrm{RS}(\mathrm{u}, \mathrm{w})$ is given as

$$
T_{d e c}=\left(2 m k+2 k^{2}\right)\left(T_{a d d}+T_{m u l t}\right)
$$

Where $\mathrm{m}=8 \mathrm{u}$ and $\mathrm{k}=4(\mathrm{u}-\mathrm{w})$ as we consider 8 -bit symbols in RS FEC codes. Moreover, $\mathrm{T}_{\text {add }}$ and $\mathrm{T}_{\text {mult }}$ are the energy consumption for addition and multiplication, respectively, of field elements in $G F\left(2^{n}\right), n=\left[\log _{2} m+1\right]$ [20]. In Micaz node one addition and multiplication of 8 bits can be performed in 1 and 2 cycles, respectively [27]. Therefore, as a result

$$
T_{\text {add }}+\mathrm{T}_{m u l t}=3\left\lceil\frac{n}{8}\right\rceil \mathrm{t}_{\text {cycle }}
$$

Where $\mathrm{t}_{\text {cycle }}$ is one cycle duration of processor, that is 250ns[6]. Based on decoding latency, the total energy consumption of RS decoding can be calculated as follows:

$$
\mathrm{E}_{d e c}=\mathrm{V} \mathrm{I}_{\text {proc }} \mathrm{T}_{\text {dec }}
$$

Where $\mathrm{V}$ is supply voltage, and $I_{\text {proc }}$ is the current for processor. Note that we assume each instruction's execution consumes approximately the same amount of voltage and current regardless of the instruction types.

\section{PERFORMANCE EVALUATION}

In this section, we study the robustness and efficiency of conventional error control protocols as a function of channel bit error rate (BER), error correcting capability, and maximum number of retransmission in WMSNs via extensive simulations. Simulation experiments are performed using ns2[11] network simulator along with a video quality evaluation tool named Evalvid[12] to analysis the performance of error control schemas in terms of energy consumption, average peak signal-to-noise ratio (PSNR), and frame loss rate. In our simulations, 50 video sensor nodes are randomly placed in 200 X $200 \mathrm{~m}$ area capable of capturing, encoding and broadcasting live video sequences to a sink node. The sender/receiver pairs are randomly chosen from a set within the area. Each node has a unique queue size of 100 and maximum transmission range of $40 \mathrm{~m}$. A CSMA/CA medium access control (802.11 MAC) is considered while RTS/CTS exchange mechanisms are disabled and end-to-end routes are established based on AODV [13]. Moreover, we use video sequence Akiyo at QCIF resolution with 300 video frames at a frame rate of $30 \mathrm{fps}$. The frames are compressed in MPEG4 format at $250 \mathrm{Kbps}$ using the FFmpeg [14] video encoder software. Also, the frames are packetized into 100-bytes video packets for energy efficiency purpose [7]. Furthermore, other simulation parameters are set based on Micaz mote hardware. All simulations are performed 20 times with different random number seeds and the results are averaged. Finally, we denote hybrid ARQ/RS error control schema with ARQ/RS(N,M) which indicate ARQ with maximum $\mathrm{N}$ retransmissions and Reed-Solomon block coded FEC; RS(M,100).

\section{A. Frame Loss Analysis}

Fig. 2, the frame loss rate is shown as a function of channel bit error rate for simple and hybrid error control schemas. Accordingly, RS $(106,100)$ results in frame loss rate that is lower than both ARQ and RS $(104,100)$. The ARQ schema with 7 retransmissions results in frame loss rate comparable to ARQ with 4 retransmissions. In particular, Fig. 2 shows that for 


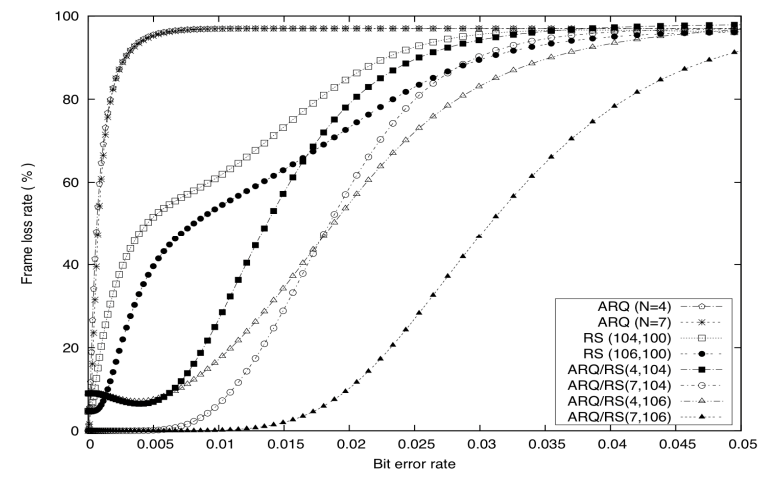

Figure 2. Frame loss rate versus channel bit error rates

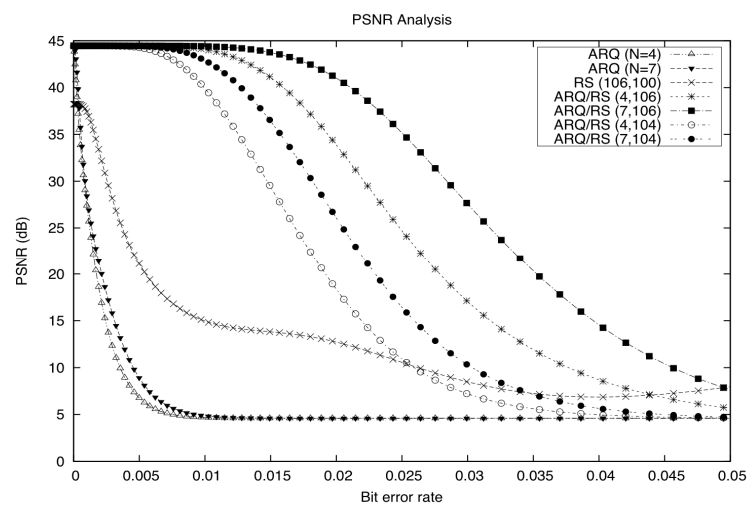

Figure 3. Average PSNR under varying channel bit error rates

ARQ $(\mathrm{N}=4)$ even a channel bit error rate of .001, leads to more than $80 \%$ loss of data. For slightly higher bit error rate of .003, the end-to-end frame loss rate of ARQ $(\mathrm{N}=7)$ is nearly $90 \%$. When BER is increased, the frame loss rate in all schemas is increased. Furthermore, it can be observed that RS codes always result in higher frame delivery rate than ARQ. The hybrid FEC/ARQ schemas exploit best features of both ARQ and FEC techniques. Fig. 2 shows using link-layer FEC remarkably reduces the frame loss rate especially at the low channel bit error rates. Also, ARQ/RS $(7,104)$ is more reliable than hybrid ARQ/RS $(4,106)$ for channel bit error rates up to .018 , while for error rates higher than these values the hybrid $\mathrm{ARQ} / \mathrm{RS}(4,106)$ schema is results in better frame delivery rate. Therefore, when hybrid schemas are considered, better reliability is provided by increasing the maximum number of retransmission at lower error rates and the strength of RS schema at higher error rates. Moreover, there is no clear winner between the RS $(106,100)$ and hybrid ARQ/RS $(4,104)$ since the first schema works better for bit error rates up to $\sim .017$ and the latter provide slightly better results for error rate higher than these values. Finally, it can be observed that the ARQ/RS $(7,106)$ schema has the best frame delivery performance among the compared schemas.

\section{B. PSNR Analysis}

In this section, we investigate the performance of different error control schemas for video delivery over WSNs in terms of PSNR. A reasonable quality is provided for the end-user if typical PSNR of a frame be more than $30 \mathrm{~dB}$. Fig. 3 shows the

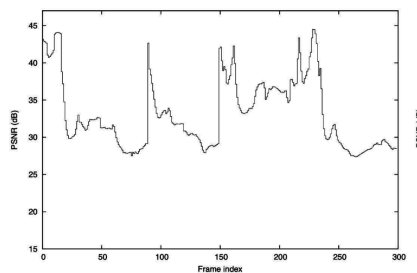

(a)

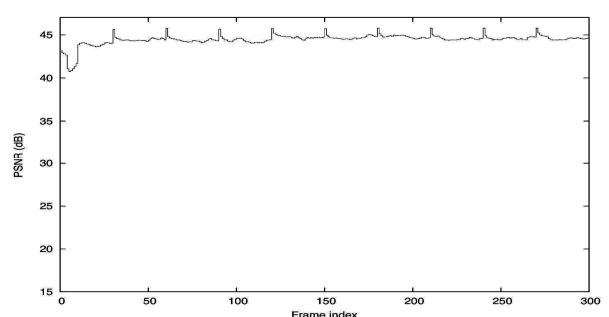

(c)

Figure 4. PSNR over 300 frames of input video for (a) ARQ, (b) FEC, (c) hybrid ARQ/FEC

average PSNR of simple and hybrid error control schemas is decreased as channel bit error rate increases. This figure shows that the average PSNR of hybrid schemas do not drop until the channel BER reaches about .01, because these schemas are sufficient for error recovery under those BER conditions. However, the average PSNR begins to drop as the BER exceeds .01. Moreover, the drop in average PSNR is more severe for ARQ and RS $(106,100)$ error control schemas. Clearly, they have not the capability to provide reasonable video quality when error rate exceeds about .0015 for ARQ, and .0003 for RS $(106,100)$. This indicates that simple error control schemas are not suitable candidates for multimedia communication over WSN. Furthermore, it is shown that hybrid ARQ/RS $(4,106)$ schema provides better PSNR than hybrid ARQ/RS (7,104). Therefore, in hybrid schemas the strength of RS has more impact on perceived video quality than maximum number of ARQ retry limit.

To investigate the video quality in more details, in Fig. 4 (a), (b) and (c), the resulting PSNR of received frames for ARQ $(\mathrm{N}=7)$, RS $(106,100)$, and hybrid ARQ/RS $(7,106)$ are shown respectively when the $\mathrm{BER}=.0007$. Fig. 4 shows that the PSNR fluctuates more significantly for ARQ and RS error control schemas. Indeed, the quality variations due to packet errors in RS $(106,100)$ are smoother than ARQ. Moreover, the PSNR level changes are minimized in the case of hybrid schema. This is very important since smoothing improves the Mean Opinion Score (MOS) of the perceived video sequence.

\section{Energy Efficiency Analysis}

The energy efficiency of the error control schemas that are discussed in this paper is shown in Fig. 5 for Micaz based wireless multimedia sensor nodes. In the figures, the average power over all sensors for simple, and hybrid schemas subject to a channel bit error rate of .03 are shown as function of their error correction capability and maximum number of retransmission. In particular, the average power consumed is calculated by dividing the total energy consumed in sensors by the total simulated time. Note that the error correction capability indicates the strength of RS schema. So for example, ARQ (N=7) with error correction capability 0 and 2 present 


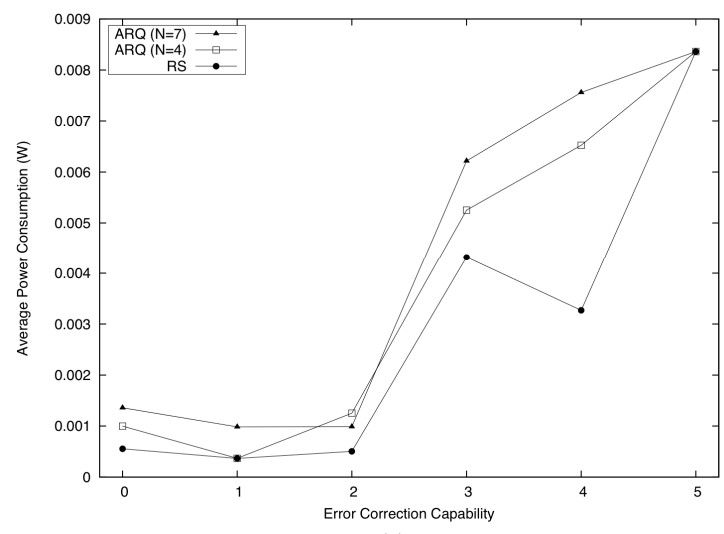

(a)

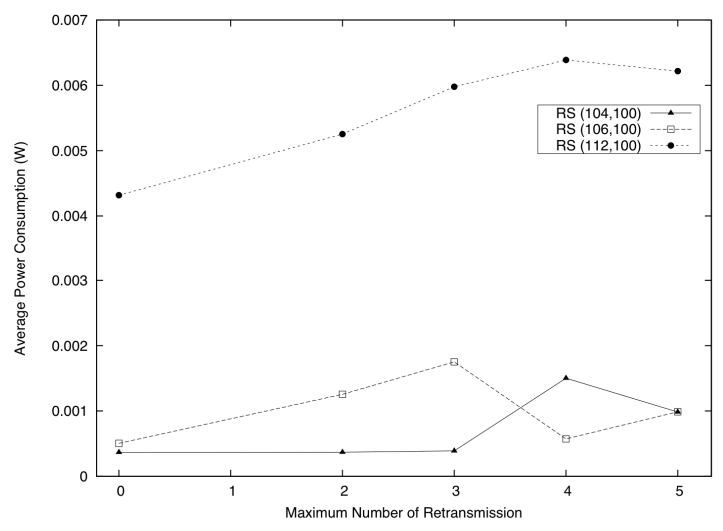

(b)

Figure 5. (a) Average power consumption versus error correction capability and (b) average power consumption versus maximum number of retransmission

simple ARQ (N=7) and hybrid ARQ/RS(7,104) schemas respectively. Fig. 5(a) shows that as the error correction capability increases, hybrid schemas increase their energy consumption. It can be observed that simple RS schema has the most efficient power consumption. Furthermore, ARQ $(\mathrm{N}=7)$ is less energy efficient compared to ARQ $(\mathrm{N}=4)$ schema. The reason is that in bad channel condition( $\mathrm{BER}=.03)$, ARQ with retry limit equal to 7 , results into several retransmission that are useless due to high channel error rate. Finally, it can be observed that for different schemas, optimum configurations can be found to minimize the energy consumption and maximize PSNR.

Fig. 5(b) illustrates the energy efficiency of discussed error control schemas as a function of maximum number of allowable retry limits. It has been shown that the error control mechanisms that use $\operatorname{RS}(112,100)$ schema result in energy consumption that is significantly higher than other error control schemas regardless of their maximum number of retransmission attempts. In particular, the $\operatorname{RS}(112,100)$ schema results in highest energy consumptions, since the energy of decoding is increased. Therefore, it can be concluded that the energy efficiency is more affected by FEC strength than retransmission attempts. Moreover, the optimum hybrid schema for RS $(104,100)$ and $\operatorname{RS}(106,100)$ can be found respectively in three and four maximum of retransmissions. Finally as shown in Fig. 5(b), the energy efficiency of reliable hybrid schemas such as hybrid ARQ/RS (N,112) is not optimized due to useless retransmissions and static FEC block coding strength.

\section{CONCLUSIONS}

In this paper, we performed simulations to comprehensively evaluate the performance of different error control mechanisms in WMSNs. It is shown that the RS schema consumes energy efficiently; however, it can not provide acceptable video quality at the receiver in higher error rates. Moreover, the ARQ schema has the worse performance in terms of PSNR. Furthermore, it can be observed from simulation results that hybrid ARQ/RS schema outperforms other schemas based on perceived video quality and frame loss rate, while it can not provide the best energy-efficient results. Therefore, energyefficient reliable multimedia delivery is the most important change that needs to be addressed by new error control protocols for WMSNs.

In the future work, we would extend this study to analysis other error control mechanisms such as erasure coding and cross-layer schemas. Furthermore, we also intend to add realtime performance metrics like delay-constrained PSNR, end-toend packet latency, and cumulative jitter to our comprehensive evaluations.

\section{REFERENCES}

[1] I. F. Akyildiz, T. Melodia, and K. R. Chowdhury, "A survey on wireless multimedia sensor networks," Computer Networks (Elsevier) J., vol. 51, no. 4, pp. 921-960, 2007.

[2] De Bardelaben, J.A.," Multimedia sensor networks for ISR applications," Signals, Systems and Computers, 2003. Conference Record of the Thirty-Seventh Asilomar Conference, Proceedings Vol. 2, pp. 2009-2012, Nov 2003.

[3] I. Akyildiz, T. Melodia, and K. Chowdhury, "Wireless multimedia sensor networks: Applications and testbeds," Proceedings of the IEEE, vol. 96 , no. 10 , pp. $1588-1605$, Oct. 2008.

[4] M. Mushkin and I. Bar-David, "Capacity and coding for the GilbertEliot channel," IEEE Trans. Infom. Theory, Vol. IT-35, No.6, PP. $1277-$ 1290, Nov. 1989.

[5] H. S. Wang, "On verifying the first-order Markovian assumption for a Rayleigh fading channel model," IEEE Trans. Veh. Technol., vol. 45, pp. 353-357, May 1996.

[6] MicaZ Datasheet. Crossbow Corp. [Online]. Available: http://www. xbow.com.

[7] Y. Sankarasubramaniam, I. F. Akyildiz, and S. W. McLaughlin, "Energy efficiency based packet size optimization in wireless sensor networks," in Proc. IEEE Int. Workshop on Sensor Network Protocols and Applications, 2003, pp. 1-8.

[8] S.Lin and D.J.Costello Jr., Error Control Coding : Fundamentals and Applications. Englewood Cliffs, NJ: Prentice-Hall, 1983.

[9] M. C. Vuran and I. F. Akyildiz, "Error control in wireless sensor networks: a cross layer analysis," IEEE Trans. ON NETWORKING, VOL. 17, NO. 4, AUGUST 2009.

[10] ATmega128 Datasheet. Atmel Corp. [Online]. Available: http://www. atmel.com

[11] Network Simulator - (ns-2), http://www.isi.edu/nsnam/ns/.

[12] J. Klaue, B. Rathke, and A. Wolisz, "EvalVid - A Framework for Video Transmission and Quality Evaluation," in In Proc. of the Inti. Conf. on Modelling Techniques and Tools for Computer Performance Evaluation, Urbana, Illinois, September 2003, pp. 255-272.

[13] C. E. Perkins, E. M. Belding-Royer, and S. Das, "Ad Hoc On Demand Distance Vector (AODV) Routing," IETF RFC 3561.

[14] F. Bellard, http://www.ffmpeg.org 